\title{
Les espaces discursifs de la frontière : mort et arbitraire dans le voyage vers l'Europe
}

Discursives Spaces of Border: Death and Arbitrariness in Journeys towards

Europe

Espacios discursivos de la frontera: la muerte y lo arbitrario en viajes hacia

Europa

\section{Anaïk Pian}

\section{(2) OpenEdition}

Journals

\section{Édition électronique}

URL : https://journals.openedition.org/remi/8728

DOI : $10.4000 /$ remi.8728

ISSN : $1777-5418$

Traduction(s) :

The Discursive Spaces of the Border: Putting Death and Arbitrariness during the Journey to Europe into Words - URL : https://journals.openedition.org/remi/10323 [en]

Éditeur

Université de Poitiers

\section{Édition imprimée}

Date de publication : 1 septembre 2017

Pagination : 45-62

ISBN : 979-10-90426-59-7

ISSN : 0765-0752

Référence électronique

Anaïk Pian, «Les espaces discursifs de la frontière : mort et arbitraire dans le voyage vers l'Europe », Revue européenne des migrations internationales [En ligne], vol. 33 - n² et 3 | 2017, mis en ligne le 01 septembre 2019, consulté le 15 avril 2022. URL : http://journals.openedition.org/remi/8728 ; DOI : https://doi.org/10.4000/remi.8728 


\title{
Les espaces discursifs de la frontière : mort et arbitraire dans le voyage vers l'Europe
}

\section{Anaïk Pian ${ }^{1}$}

\begin{abstract}
Dans un monde à la fois globalisé et profondément fragmenté, les formes prises par les frontières contemporaines font l'objet de réflexions renouvelées. Plusieurs travaux récents sur les migrations internationales manifestent un intérêt marqué pour les concepts de border, boundary (De Genova, 2005), borderland (Bliber Coutin, 2003 ; Agier, 2014), borderities (Amilhat-Szary et Giraut, 2015), borderscape (Rajaram et Grundy-Warr, 2008 ; Brambilla, 2014) ou encore border(land)scape (Ferrer-Gallardo et al., 2016). Issus de la terminologie anglo-saxonne, ces concepts sont mobilisés dans des études s'intéressant aux effets des politiques migratoires sur la production de nouveaux espaces-frontières, qu'il s'agisse d'évoquer des lieux fermés (dont les centres de rétention sont un archétype $)^{2}$, des campements informels, des marges urbaines ou plus largement de vastes territoires traversés par des frontières à la fois visibles et invisibles, où l'enfermement se vit " à ciel ouvert ". Étymologiquement issu du substantif "front " (Foucher, 1998), le terme frontière se décline de deux façons en anglais : border, relevant d'une conception géographique et territoriale, réfère à une démarcation entre espaces tandis que le terme boundary fait plutôt référence à des frontières sociales et symboliques créant une distinction eux/nous. S'intéressant au fonctionnement des centres de rétention sur l'île italienne de Lampedusa, Tassin (2013) invite à penser ensemble le couple "border/boundary " ${ }^{3}$ afin de saisir la manière dont le contrôle des populations indésirables s'appuie et fabrique tout à la fois des processus de catégorisations des individus et de lieux. Dans le champ des migrations internationales, Agier (2014) utilise le terme de borderland - ou paysage de frontières - pour saisir, d'un point de vue ethnographique et dans une perspective articulant le local et le global à une échelle mondialisée ${ }^{4}$, la multiplication et densité des " situations de
\end{abstract}

\footnotetext{
1 Maître de conférences en sociologie, Université de Strasbourg, Le Patio, 22 rue René Descartes BP 80010, 67084 Strasbourg Cedex; pian@unistra.fr

2 Même si ces derniers peuvent aussi être analysés comme des espaces de circulation (Makaremi et Kobelinsky, 2009).

3 Voir également sur cette articulation Fassin (2011).

4 Dans une perspective s'intéressant à l'autorité politique, le terme " borderland » réinterroge les relations entre centre (d'un État) et périphéries (Cusco, 2016) avec une importance donnée aux confins et aux marges, tout en incluant dimensions politiques et symboliques.
} 
frontières $\aleph^{5}$. En s'inspirant des approches de Foucault sur la gouvernementalité et le pouvoir, Amilhat-Szary et Giraut (2015) proposent de parler de borderities terme que les auteurs traduisent par "frontiérité " - afin de " mieux exprimer les dimensions individuelles et collectives de ce qui se joue dans le rapport aux limites internationales et à leurs franchissements "(Amilhat Szary, 2015 :108) tout en prenant en compte le point de vue de ceux qui traversent ou tentent de traverser les frontières. Mis en avant par Rajaram et Grundy Warr (2008), le concept de borderscape est repris par Brambilla (2014) pour rendre compte du processus déterritorialisé, dispersé et mobile des frontières actuelles ainsi que leurs aspects performatifs. L'auteure applique ce concept à une nouvelle géographie des frontières entre la Libye et I'Italie appréhendée en termes de oasis scapes, de camp-scapes et de business-scapes.

En dépit de leurs nuances ${ }^{6}$, ces concepts invitent à décrire et analyser un mode particulier de gouvernance des migrations ainsi que les implications qui en découlent sur les espaces vécus des migrants (Pian, 2016) ${ }^{7}$ : espaces pratiqués et représentés, mais aussi (ra)contés. Dans le cadre de cet article, les frontières seront avant tout appréhendées sous l'angle des discours issus de la confrontation à l'expérience de leurs franchissements (ou tentative de franchissements). Ces expériences de la frontière, qui génèrent des pratiques discursives, peuvent être vues comme le point de crispations entre "frontières non indexicales " nonindexical border - et " frontières indexicales " - indexical border - (Green, 2012) : le premier terme réfère ici aux frontières matérialisées par les dispositifs de contrôle ; le second renvoie aux frontières comme lieu indexical dont le sens dépend d'un contexte donné, et qui sont relatives au point de vue d'où l'on se place - et d'où l'on parle.

Dans cette perspective, en mobilisant différents terrains menés auprès de migrants cherchant à rejoindre clandestinement l'Europe (Pian, 2009a, 2010 et 2016), l'article s'intéresse à la fabrique des discours sur la mort et, partant, sur la violence aux et des frontières. Une attention particulière sera portée sur les mises en mots de ce qui est perçu et vécu comme un arbitraire institutionnel ${ }^{8}$. Dans le sens courant, le terme arbitraire revêt deux significations : il désigne d'une part une action " qui résulte de la volonté et du bon plaisir d'une personne "; il réfère d'autre part à une pratique " en violation de la loi ou de la justice " (définitions du dictionnaire Larousse). D'un point de vue sociologique, I'arbitraire apparaît indissociable des rapports de pouvoir et de domination dans lesquels il s'inscrit. Soulevant l'application différentielle du droit ou de la loi, il interpelle la " gestion des illégalismes " (Fischer et Spire, 2009) qui renvoie au " pouvoir discrétionnaire [des] agents [...] mandatés par l'État pour prendre des décisions susceptibles de bouleverser la vie des sujets qu'ils ont en face d'eux". Ce pouvoir, précisent les auteurs, s'exerce à partir d' " un répertoire d'actions qui va de la sanction à la transaction, et qui ne saurait se réduire à la manifestation

5 Voir également Puig et al. (2014).

6 Celles-ci ne peuvent être explicitées ici, ce qui nécessiterait un développement à part entière.

7 Sur la notion d'espace vécu, voir aussi Séchet et Veschambre (2006).

8 Si nous n'entrons pas dans les détails d'une analyse de discours telle que la pratique des linguistes, l'approche proposée tente de dépasser les limites d'une stricte analyse de contenu omettant les contextualisations socio-politiques. 
autonome du pouvoir étatique " (Ibid.). Toutefois, I'objet ne sera pas tant ici, à l'instar d'un récent numéro de Cultures \& Conflits (2015), de s'intéresser à la violence des pratiques aux frontières ; il s'agira plutôt, de manière complémentaire, d'orienter le regard vers les discours des migrants ainsi suscités, qu'ils soient tenus au chercheur (en position d'extériorité initiale dans le cadre d'une enquête par familiarisation) ou au groupe de pairs.

Le matériau mobilisé relève d'une analyse secondaire des données, ce " reste " (Beaud et Weber, 2003) mis de côté de I'enquête ethnographique. II a été recueilli lors de plusieurs terrains (combinant observations, discussions informelles et entretiens enregistrés). Ces derniers ont été réalisés en 2008 au Sénégal et entre 2003 et 2009 au Maroc ${ }^{9}$. L'enquête au Sénégal a été menée auprès de refoulés ayant tenté la traversée vers les îles Canaries (puis placés jusqu'à leur refoulement en centre de rétention) et rencontrés par la suite à Dakar, Mbour et Cayar dans leurs lieux de vie quotidienne (travail, quartier, maison familiale, etc.) ; l'enquête au Maroc a principalement été effectuée dans trois villes marocaines et leur périphérie, à Casablanca, Rabat et Fès auprès de Sénégalais cherchant à rejoindre $\mathrm{I}^{\prime} \mathrm{Europe}^{10}$. Les terrains sont donc multi situés " entre sites ", mais aussi en termes de suivi de trajectoires"1. Des enquêtés, en effet, ont été rencontrés à plusieurs reprises dans différentes villes du Maroc, mais aussi dans leur pays d'origine suite à leur retour au Sénégal (par rapatriement ou non). Ont ainsi été recueillis les témoignages de migrants ${ }^{12}$ sur leur expérience migratoire et, partant, sur leurs confrontations aux frontières externes de l'Europe et aux acteurs institutionnels les incarnant. Revenant sur l'étymologie du mot " témoin ", Fassin (2010) rappelle les deux origines latines du terme. La première, "superstes ", fait référence au témoin qui a vécu l'évènement et qui lui a survécu tandis que la seconde, " testis ", désigne l'observateur extérieur d'une scène, qui y a assisté sans l'avoir personnellement vécue. Ici, c'est bien en tant que " superstes " que la parole des migrants est prise en compte.

9 Entre 2003 et 2007, le terrain marocain a été mené dans le cadre d'un doctorat de sociologie. Il a été poursuivi en 2009 dans le cadre du projet européen Eurobroadmap sur les représentations de I'Europe dans le monde (URMIS/RIATE/ITC, Paris 7). Le terrain au Sénégal a été effectué dans le cadre de l'ANR "MITRANS Migrations de transit en Afrique : dynamiques locales et globales, gestion politique et expériences d'acteurs ", URMIS-SOLIIS-Institut Français d'Afrique du Sud.

10 Dans les deux cas, le corpus est majoritairement constitué d'hommes âgés entre vingt et quarante ans. Le nombre total d'entretiens n'a pas été précisément comptabilisé, mais plus de quatre-vingts personnes ont été rencontrées. L'approche défendue est celle de l'entretien ethnographique qui, se démarquant " du joug de la pensée statistique " (Beaud, 1996 : 234), privilégie l'entretien comme " situation d'observation " (Ibid.). Pour plus de détails sur les conditions de terrain, voir Pian (2009a).

11 Sur la démarche multisite, voir Marcus (2010).

12 Les Sénégalais rencontrés au Maroc alors qu'ils cherchaient à passer clandestinement en Europe revendiquaient I'appellation " aventuriers " - tout en s'y démarquant à partir du moment où ils renonçaient au passage (Pian, 2009a). En revanche, les Sénégalais rencontrés au Sénégal, partis en pirogues des côtes sénégalaises pour rejoindre les îles Canaries, ne se considéraient pas comme des " aventuriers". Pour eux, le terme s'appliquait à ceux faisant un long parcours par étapes (via le Maroc, la Libye, etc.), pouvant prendre plusieurs années, pour tenter de rejoindre l'Europe. Sur les rapports sociaux s'exprimant à travers les usages des auto et hétéro-définitions du terme "aventurier ", voir Pian (2009a) et I'article de Canut dans ce numéro. 
L'article est structuré en deux parties. La première identifie différents registres de discours sur la mort, registres faisant référence à une mort physique au sens propre ainsi qu'à une mort sociale au sens figuré. La seconde partie s'intéresse à la manière dont la confrontation à une gestion institutionnelle opaque des migrations - notamment en centre de rétention - alimente certaines rumeurs, celles-ci pouvant être appréhendées comme un "fait social " (Bonhomme, 2009) témoignant, entre autres, d'un rapport aux institutions.

\section{Du slogan à l'expérience vécue}

Le slogan Barça wala Barsakh (Barcelone ou la mort ou l'au-delà en wolof), brandi par de nombreux Sénégalais ayant tenté en 2006 -2008 de rejoindre les îles Canaries depuis les côtes sénégalaises (ou se disant prêts à le faire), montre que la référence à la mort est bien présente ${ }^{13}$ dans les discours des migrants empruntant la voie de la traversée clandestine. Pour rappel, pour la seule année 2006, 31000 personnes atteignent les îles Canaries en pirogue. Ce chiffre masque quelques milliers de morts et disparus en mer dont l'absence de dénombrement précis n'enlève rien aux marques laissées dans les localités très touchées par les départs ${ }^{14}$. Comme le souligne un pêcheur rencontré à Mbour en 2008 après son refoulement des îles Canaries : "On dit que ceux qui sont morts dans la mer clandestin, $c^{\prime}$ est au moins autant que le Diola. ${ }^{15}$ Les discours évoquant la mort ou le risque de mort dans ces routes vers l'Europe comportent toutefois de profondes nuances selon qu'il s'agisse de légitimer, justifier voire de rationaliser l'émigration, de faire part d'expériences extrêmes confrontant aux obstacles naturels ou de décrire les formes de violences institutionnelles vécues dans le cours du périple effectué.

\section{La mort sociale versus la mort physique}

À un premier niveau de discours, l'expression Barça wala Barsakh est érigée en emblème de la détermination à défier les frontières de l'Europe. Derrière ce défi, l'enjeu est d'exister socialement face à une vie qui, en l'état, est dite ne valant rien. Comme le dit Malik originaire d'une banlieue de Dakar : "Je ne suis pas parti pour partir, mais pour ne pas rester " (étudiant, vingt ans, dont le père est pêcheur) ${ }^{16}$. Ici, la prise de risque effectuée en empruntant la voie des pirogues est énoncée comme un moyen de s'opposer ou du moins de refuser la mort sociale. Celle-ci englobe le manque de considération des proches, l'impossibilité d'honorer ce qui relève du devoir, d'apporter une contribution financière à la vie familiale, bref, d'exister socialement (Timera, 2001 ; Pian, 2011a) dans

13 Même si elle peut être tournée en dérision, comme le montre la contribution de Kobelinsky dans ce numéro.

14 Voir par exemple les travaux de Bouilly (2008) pour le cas deThiaroye-sur-Mer, commune de la ville de Pikine dans la région de Dakar.

$15 \mathrm{Ce}$ qui est communément appelé le " pays diola " se situe en Casamance et plus particulièrement en Basse-Casamance, région située au sud du Sénégal. Les diolas constituent le groupe ethnique majoritaire de la Basse-Casamance, mais se retrouvent aussi notamment en Gambie et en Guinée-Bissau. À ce sujet et pour plus de détails sur les enjeux politiques y afférant, voir Foucher (2003).

16 Sur la diversité des profils sociologiques des Sénégalais ayant tenté la traversée clandestine depuis les côtes sénégalaise, voir Pian (2011a). En I'absence d'incidents, la durée de traversée est estimée à une dizaine de jours. 
un contexte de crise économique et plus largement de reconfigurations des rapports familiaux au Sénégal.

Risquer sa vie, et même la perdre, c'est alors " entrer en action " et montrer aux yeux de tous que l'on est "capable " pour reprendre les propos de ce jeune Sénégalais ${ }^{17}$. La mort physique, si elle doit advenir, est dite comme un moyen d'accéder à une reconnaissance sociale post-mortum. Ces défis, lancés tout à la fois à la mort et à la vie, s'accompagnent de métaphores empreintes de références religieuses : dans les récits recueillis, I'évocation du SIRAT est mobilisée à plusieurs reprises pour rendre compte de la dangerosité des courants maritimes au large des côtes marocaines et, ce faisant, de l'épreuve fatidique à affronter pour atteindre les îles Canaries. Comme le souligne ce fils de pêcheur âgé d'une vingtaine d'années, refoulé des îles Canaries et sans emploi (avant et après sa tentative de départ) :

"Car à ce moment-là, les clandestins sont devenus des artistes. Tu sais, dans le Coran, on dit que pour aller au paradis, il y a un chemin qu'on doit passer qu'on appelle le SIRAT. Nous, on appelle ça le SIRAT. Car le Coran dit qu'on doit passer en marchant sur un fil très mince. Donc les clandestins appellent la traversée SIRAT, et le Sénégal le monde des problèmes, I'Europe, le paradis, et pour cela, il faut passer par SIRAT. SIRAT, c'est le chemin à partir du Maroc où tous les clandestins commencent à paniquer car c'est plus houleux..."

Par cette allégorie, il est fait référence à la traversée du pont lors du jugement dernier. Dans le Coran, ce pont est décrit comme " plus tranchant que l'épée " et " plus fin que le cheveu ". Il est écrit que les non-musulmans ne parviendront pas à le traverser et tomberont en enfer ; les musulmans ayant pêché glisseront d'abord en enfer, puis, après un châtiment, pourront rejoindre le Paradis. Cet exemple montre comment, face au risque de mort inhérent à la traversée clandestine, tout un univers de signes et de symboles est convoqué ${ }^{18}$ mobilisant à la fois le registre religieux et les représentations de l'Europe : ici, celle-ci est associée à un Paradis dont l'accès, parsemé d'obstacles, nécessite la traversée d'un passage transitoire dangereux marquant une frontière entre deux mondes. Le franchissement de cette frontière est associé à une véritable épreuve avec des héros glorieux, déchus et/ou disparus.

L'assurance affichée face à une mort qui n'est pas crainte, mais défiée s'effrite toutefois lorsqu'est mise en mots l'expérience d'une confrontation imminente et non plus virtuelle à la mort physique. Cela ne veut pas dire que parmi les discours tenus au chercheur, certains seraient plus vrais que d'autres. En revanche, si les discours sont toujours cadrés ${ }^{19}$ selon leur contexte de production et les interlocuteurs en présence, ils le sont aussi selon le moment et la focale du récit. Les discours laissant place à une appréhension de la mort émergent lorsque les

17 Le risque perçu est fréquemment minimisé par les pêcheurs aguerris qui mettent en avant leur expérience de pêche au long cours, impliquant des sorties en mer s'étendant sur plusieurs jours.

18 Univers dont les rappeurs sénégalais se sont également saisis : ces derniers, en effet, se sont démarqués par une série de productions mettant en garde contre les dangers de l'immigration clandestine tout en dénonçant les politiques migratoires européennes ainsi que la position tenue par le gouvernement sénégalais. A ce sujet, voir Moulard-Kouka (2014).

19 Nous nous référons ici à la notion de cadrage des discours (Cefai, 2001). 
Sénégalais rencontrés évoquent rétrospectivement le naufrage de leur embarcation et/ou la dureté des conditions de traversée (affronter la chaleur ou la pluie, abrité au mieux sous une bâche ; faire face au manque d'eau et de nourriture ; se rendre compte avec effroi que la pirogue est en train de se fissurer, assister aux délires de compagnons de route, etc.). En témoigne le récit de la traversée vers les îles Canaries fait par ce pêcheur de Mbour, âgé de trente-huit ans et travaillant, comme son père et ses grands-parents avant lui, dans le secteur de la pêche depuis son plus jeune âge :

"La pirogue prenait l'eau, il fallait écoper. Les gens ne voulaient plus porter de gilets de sauvetage, ils disaient que mourir dans la mer après 10 minutes ou 40 minutes, c'est la même chose."

De la même façon, Mbalo (trente ans), travaillant avant son départ comme ouvrier journalier sur des chantiers de construction, souligne à propos de camarades d'infortune ayant emprunté la même pirogue que lui : "Avant de voir leur mort, ils se sont jetés dans la mer pour être tranquilles ". Assister, désarmé, au décès de compagnons de route dont les corps sont jetés par-dessus bord est également raconté comme une épreuve particulièrement éprouvante :

"C'est horrible, tu vois que tu n'es rien et tu te demandes qu'est-ce que c'est la vie... Tu n'es rien, tu meurs comme ça, peut-être que ton corps va être retrouvé sur une plage, anonyme... " (Sy, mécanicien, ayant tenté de rejoindre les îles Canaries à partir du Maroc)

Ce qui s'exprime à travers ces propos, c'est un sentiment d'impuissance conjuguant fatalisme (cela ne sert rien de mettre un gilet de sauvetage) et nihilisme (la vie ne semble plus avoir de sens). Mais ce n'est pas seulement le face-à-face avec les forces naturelles qui invite les migrants à moduler la teneur du slogan Barça wala Barsakh ; c'est également, dans un registre complémentaire ${ }^{20}$, la confrontation à un pouvoir discrétionnaire.

\section{Un pouvoir discrétionnaire, détenteur de vie et de mort}

La référence à la mort intervient souvent lorsque les intéressés racontent comment, à un moment de leur expérience migratoire, ils se sont retrouvés face à des agents de l'État (militaires, gardes-frontières, policiers, etc.) ou " professionnels de la frontière " (Lask, 1995) incarnant l'ordre étatique et qui, par un seul geste, une seule décision, un seul mot d'ordre peuvent ou non les maintenir à la frontière de la vie.

C'est le cas d'Amadou, rapatrié au Sénégal au lendemain des évènements de Ceuta et Melilla en 2005. Amadou est arrivé au Maroc en 2001, dans I'espoir de rejoindre l'Espagne. II se reconvertit finalement dans le commerce de rue à Casablanca. En 2005, alors qu'il vendait sa marchandise dans les rues de la

20 À cet égard, voir également les travaux de Shindel (2016) mettant en relation (tant d'un point de vue pratique que discursif) les nouvelles technologies de contrôles aux frontières européennes et l'exposition accrue aux dangers et aléas de la nature auxquels se confrontent les étrangers cherchant à rejoindre clandestinement l'Europe. 
Médina, il est arrêté par les forces de l'ordre marocaines dans le cadre d'une vaste opération de rafle, puis expulsé à la frontière algérienne. II fait partie de ces migrants ayant erré, plusieurs jours, dans le no man's land désertique entre le Maroc et l'Algérie avant que, face au tollé international suscité, les autorités marocaines et algériennes ne se décident de revenir chercher dans le désert les migrants expulsés, pour ensuite organiser des rapatriements à destination des pays d'origine. Comme le souligne Agier (2013:68) en faisant référence aux enquêtes menées par Lecadet (2012) auprès d'expulsés maliens, l'expulsion "fait exister la frontière par son dehors " et instaure un tri entre ceux qui sont autorisés ou non à vivre dans le " dedans " de la frontière. La violence du processus - pour les expulsés - est renforcée par la manière dont elle est mise en acte. Rencontré au Sénégal plusieurs mois après son rapatriement du Maroc, Amadou raconte ainsi son expulsion :

"On nous a amenés à Fès, là, on a enfoncé dans le bus d'autres gens et on nous a conduit à Oujda [...]. Un camion nous a conduits jusqu'au désert. Personne ne se révolte. Les policiers étaient armés et si on ripostait, ils pouvaient te tuer là, comme ça, comme une goutte d'eau, sans que personne ne sache."

Après avoir erré plusieurs jours dans le désert, tour à tour refoulés par les gardes-frontières algériens et marocains, Amadou atteint, avec un petit groupe de compagnons de route, un village de l'Est marocain. Exténué, déshydraté, le petit groupe s'adresse à des Marocains pour leur demander du pain et de l'eau lorsqu'arrive une patrouille de police. Amadou rapporte les propos alors tenus :

"Il vaut mieux que vous nous tuez là, sur place, plutôt que de nous laisser agoniser [...]. Pourquoi vous nous avez jetés dans le désert? Si vous ne voulez pas de nous, il faut nous retourner chez nous, mais pas nous faire ça. On est des hommes,

on a une famille, on n'est pas un animal. "

Ce récit fait au chercheur exprime plusieurs rapports à l'ordre institutionnel. La revendication d'une humanité niée " on est des hommes " s'oppose au traitement infligé, traitement renvoyant au statut "d'homo sacer ", celle de I'homme dont il ne reste plus que la "vie nue " (Agamben, 1998) face à des autorités détenant un pouvoir de vie et de mort ${ }^{21}$. Les propos tenus font part un écart ou décalage entre ce que l'État fait des corps ("enfoncés", réduits à une "goutte d'eau ", à un " animal ") et ce que l'on est, estime être. Qui plus est, le pouvoir ainsi exercé prend place dans une situation d'anonymat. Ce qu'exprime le discours d'Amadou, en effet, c'est la vision d'une mort dans l'indifférence générale, mais aussi d'une mort donnée en toute impunité par des agents de I'État qui n'en seront pas inquiétés ("ils pouvaient te tuer là, comme ça, sans que personne ne le sache "). À travers ces mises en mot, ce sont des zones d'infradroit (Lochak, 1985) qui sont décrites et vécues comme telles. Lochak distingue deux types de droit, l'un objectif, I'autre subjectif, censés fonctionner ensemble et de manière dialectique dans un État de droit. Si les règles en vigueur (droit objectif) sont un instrument du pouvoir, dans le même temps note l'auteure, les individus doivent avoir la possibilité de mobiliser le droit en vue d'obtenir une protection contre les excès voire l'arbitraire du pouvoir (droit subjectif). Or,

21 Sur les violences policières commises, voir par exemple les rapports de Médecins Sans Frontières (2005 et 2013). 
constate Lochak, le régime appliqué aux étrangers se situe très souvent du côté de l'État de police dans la mesure où le droit subjectif tend à disparaître, tandis que le droit objectif est confié à l'arbitraire des autorités administratives ${ }^{22}$.

\section{Dire la « vie nue ", entre folie et résistances}

Dire la " vie nue " pour ceux qui en ont fait l'expérience, c'est raconter un "évènement " au sens où l'entend Leclerc-Olive (1997 : 20). Pour l'auteure, l'évènement est " ce qui fait irruption, discordance, qui introduit un changement, qui marque une discontinuité " dans une trajectoire biographique. Mais dire la " vie nue " c'est aussi, du moins parfois, tenter d'y résister par la parole.

\section{La mortification des corps}

La mise à l'épreuve d'une humanité niée est prégnante dans plusieurs récits évoquant l'arrivée aux îles Canaries puis le placement en centres de rétention. Certes, dans un premier temps du moins, le repérage par les patrouilles de la Guardia Civil est décrit comme un soulagement lorsque les embarcations de fortune arrivent à destination : la traversée se termine ; une étape du voyage est franchie. À l'instar des intervenants de la Croix Rouge qui, très vite, les prennent en charge, les membres de la Guardia Civil peuvent être perçus comme des acteurs qui "sauvent ", participant d'une prise en charge humanitaire où la reconnaissance de l'autre, en tant qu'être humain, est prise en compte ${ }^{23}$. Ces propos cèdent vite la place, toutefois, aux récits d'expériences dépersonnalisantes et humiliantes : déshabillage, fouilles au corps ; regroupement et entassement derrière des barrières de sécurité ; interrogatoire de police, etc. Autant de pratiques auxquelles s'est intéressée la littérature sociologique sur les lieux d'enfermements : pour ne citer que quelques exemples, on peut se référer au célèbre ouvrage de Goffman (1968) sur l'asile psychiatrique, aux travaux de Boe (2015) sur les conditions de détention des étrangers en prison en France et aux

\footnotetext{
22 Plusieurs études ethnographiques invitent à prendre en compte un autre aspect de la violence institutionnelle. À I'absence ou au non-respect du droit (enfermement et/ ou refoulement arbitraires, etc.) s'ajoute parfois un " trop-plein " du droit dès lors que la multiplication et juxtaposition - aux échelles nationales et internationales - de lois, de règles et de conventions posent un cadre juridique très complexe qui échappe aux concernés (même assistés d'avocats). À cet égard, les travaux de Darley (2009) sur des centres d'enfermement en République Tchèque et en Autriche ou ceux de Clochard (2014) sur la prison de Nicosie à Chypre montrent bien la complexité du " pouvoir administratif légal hypertrophique " (Makaremi et Kobelinsky, $2009: 21$ ) auxquels se heurtent les étrangers confrontés aux institutions chargées de leur mise à l'écart.

23 Par comparaison, les membres de Guardia Civil chargés de la surveillance des enclaves espagnoles de Ceuta et Melilla situées dans le nord du Maroc sont perçus d'une manière beaucoup plus négative (cf. Pian, 2013 et la contribution de Kobelinsky dans ce numéro). Ces différences s'expliquent notamment par les exactions et, plus largement, les "refoulements à chaud " vers le Maroc commis en cette zone frontalière. Ces pratiques sont régulièrement dénoncées par les associations de défense des droits des migrants. Voir par exemple : Cimade et AFVIC (2005) Refoulements et expulsions massives des migrants et demandeurs d'asile : récit d'une mission de I'AFVIC et de la Cimade, 9 p., [en ligne]. URL : http://www.algeria-watch.org/pdf/pdf_fr/afvic cimade121005.pdf. Voir également : Migreurop et Gadem (2015) Ceuta et Melilla, centres de tri à ciel ouvert aux portes de l'Afrique?, [en ligne]. URL : http://www.migreurop.org/ article2666.html. On peut aussi, à ce sujet, voir : Comité européen pour la prévention de la torture et des peines ou traitements inhumains ou dégradants (2015) 25e rapport général du CPT, Conseil de I'Europe, 80 p., [en ligne]. URL : https://rm.coe.int/1680696acc
} 
États-Unis ou encore à ceux de Makaremi (2009) décrivant, dans le contexte français, les techniques de gestion et de rationalisation des corps à expulser ${ }^{24}$.

Kader est parti au Maroc en 2002 dans l'idée d'être recruté dans une équipe de foot marocaine, tout en y poursuivant ses études. Ses projets bifurquant, il tente la traversée vers les îles Canaries depuis le Sahara marocain. Renvoyé au Maroc puis contrôlé en situation irrégulière plusieurs mois plus tard sur le territoire marocain, il est sommé de quitter immédiatement le pays s'il ne veut pas être expulsé à la frontière algérienne. Désabusé, il décide de rentrer au Sénégal. Je le rencontre par l'intermédiaire d'Amadou qu'il a connu au Maroc. Nous convenons d'un rendez-vous chez lui, dans la banlieue de Dakar ; mais, à mon arrivée, Kader se montre hésitant, ne voulant plus revenir sur son passé, de peur dit-il, de raviver des plaies. II accepte finalement de me parler, et nous restons près de 3 heures à discuter, dans sa chambre. À la fin de l'entrevue, il me remercie pour l'avoir écouté sans le juger. Kader raconte le moment où son embarcation est interceptée par les gardes-côtes à proximité des îles Canaries. II revient sur son transfert en bateau vers le centre de rétention de Lanzarote et l'attente subie, à son arrivée sur l'île, derrière un " cordon rouge et blanc " : " c'est les barrières qu'on met pour délimiter une zone d'accident " explique-til. Perçues comme humiliantes, ces barrières symbolisent à ses yeux la mise à l'écart des parias. Elles marquent une "clôture sociale " (Fischer, 2005) instituant une rupture avec I'ordre des interactions ordinaires. Kader dit alors souffrir du regard des gens qui s'attroupent, des "curieux ", des journalistes et cameramen qui les attendent sur place. II explique porter les mains à son visage de peur d'être reconnu au journal télévisé par une connaissance depuis le pays d'origine. Se donne ainsi à lire un contraste entre d'un côté un traitement anonyme et de l'autre, la profusion de regards, de clichés qui violent l'intimité. À moins que cette violation de l'intimité ne participe de cette anonymisation à laquelle ces migrants sont renvoyés de manière forcée : "Là-bas (dans les centres de rétention) on n'est rien, que des immigrés clandestins " ajoute Kader.

Lors de nos différentes rencontres à Saly, où il a trouvé refuge auprès d'amis ayant, eux aussi, tenté la traversée vers les îles Canaries, Thiarnow (vingt-six ans, marié, sans enfant, diplômé d'un BTS commercial) met en mots une expérience pour partie similaire. Quelques jours après son départ de Saint-Louis au Sénégal, son embarcation part à la dérive puis est interceptée par les gardes-côtes mauritaniens. Faisant part des mauvais traitements reçus en centre de rétention (il sera par la suite expulsé à la frontière sénégalaise), il insiste : " c'est comme si tu étais un sac vide ". Exprimant l'inégale valeur des vies inscrites dans des rapports de pouvoir et de domination, les expressions " n'être rien " ou " être un sac vide " reviennent de manière récurrente dès lors qu'il s'agit d'évoquer le rapport aux agents incarnant la surveillance des frontières. À son retour au Sénégal, Thiarnow est hospitalisé en psychiatrie durant quelques semaines. La violence, telle qu'elle est dite, c'est aussi la dépossession d'une identité, " pour soi et pour autrui " (Dubar, 1991) et qui peut profondément marquer les corps, comme le montrent plus largement Petit et al. (2014) à propos des effets de la migration internationale sur la santé mentale des migrants au Sénégal.

24 Plus largement, sur la manière dont les rhétoriques humanitaires et sécuritaires se mêlent dans la gestion des migrations, voir la réflexion de Cuttitta (2015) à partir du cas de Lampedusa. 
Ces discours sur la " mortification " des corps, pour reprendre une expression goffmanienne, doivent cependant être resitués : d'une part, comme souligné précédemment, ils constituent une partie seulement des discours tenus qui, à d'autres moments, mettent plutôt en avant l'image d'individus acteurs de leur destin (Pian, 2009a ; Canut, dans ce numéro) ; d'autre part, ils n'enlèvent rien, dans la pratique, à la mise en place d'actes de résistances. Ces actes peuvent prendre la forme de tactiques ou micro-tactiques visant à résister à la " vie nue " du confinement, comme le décrit Clochard (2016) à propos des étrangers placés en rétention administrative dans différents pays d'Europe. La parole, en tant qu'" action et praxis sociale " (Canut et Sow, $2014: 11$ ) prend également ici toute son importance. Certains tentent de résister en revendiquant publiquement des droits - à l'image de refoulés au Sénégal (Pian, 2011b) ou de migrants "bloqués " au Maroc, comme le montre le témoignage de Fiston Massamba recueilli par Claire Rodier (Massamba, 2006). Mais une autre dimension discursive, encore, est à relever : elle concerne la production de rumeurs "énoncé[es] en contexte qui implique[nt] des évènements, des gestes et des affects" (Bonhomme, 2009:30)25 par lesquelles les intéressés tentent de résister à une incertitude qui les dépasse, même si, dans un même mouvement, ces rumeurs génèrent tout à la fois elles-mêmes des incertitudes. C'est de ce dernier point dont il va être question.

\section{Faire face à l'arbitraire : production et circulation de rumeurs}

À partir d'une enquête ethnographique au centre CETI (Centre de séjour temporaire pour immigrés) de Ceuta, Andersson (2014) insiste sur le fait que le maintien dans l'attente constitue, en tant que " management temporel ", un mode important de gestion et de contrôle des frontières européennes. Pour l'auteur, les frontières forment une géographie et un paysage temporels complexes où la rapidité des interventions des États appuyées par une technologie avancée (interceptions des embarcations, des migrants tentant de franchir des frontières délimitées par des barbelées) participent à créer en retour, pour les migrants, des expériences de lenteur et d'attente. De la même façon, à partir de ces travaux sur les demandeurs d'asile et les étrangers en centres de rétention au Royaume-Uni, Griffiths (2014) met en évidence différentes formes subjectives de l'attente au regard des possibles projections temporelles des intéressés. De telles expériences se retrouvent dans les récits des migrants en centre de rétention aux îles Canaries. Le récit de Cheir rapporté ci-dessous rejoint l'expérience du " sticky time " décrit par l'auteure pour rendre compte de la durée et de la lenteur d'une attente devant aboutir à un changement de situation :

"On attend, la journée, et même la nuit... Les Espagnols nous on donnés des chaussures en plastique, du dentifrice, un peu de lessive... On nous apporte deux repas par

jours... Régulièrement, un policier vient avec une liste d'une dizaine de noms... Selon les bruits de couloir, on dit que si tu es interpellé la nuit, tu vas être rapatrié, si c'est le jour, peut-être que l'on va t'emmener en Espagne. " (Pêcheur, trente ans, refoulé au Sénégal en 2006)

25 L'auteur distingue la rumeur de la " croyance " en ce que cette dernière serait plus profondément ancrée " supposant davantage une persistante de la représentation au sein du groupe " (Ibid. : 31). 
Mais ce témoignage ne fait pas seulement état d'une incertitude temporelle ; il met en exergue une double incertitude temporelle et spatiale concernant autant le moment que le lieu du transfert effectué par les autorités. Ces lieux d'enfermement fonctionnent sur un rapport asymétrique à l'information : l'institution prélève des informations sur les corps, mais n'en donne pas. Dès lors, les migrants se raccrochent aux rumeurs à partir desquelles ils espèrent, désespèrent, se mettent à douter : comme l'explique Cheir un peu plus haut, selon le moment de la journée où les agents viennent les chercher, la durée de leur rétention, les intéressés pensent qu'ils vont être rapatriés ou non. Dans un ouvrage consacré à une sociologie politique des rumeurs, Aldrin (2005) note comment l'absence d'informations officielles est propice à leur développement, et ce, que les rumeurs soient fausses ou avérées. Dans son étude sur les " bembas ", rumeurs alimentant le quotidien des prisonniers politiques sous la dictature en Argentine (1976-1983), Ípola (2006 : 174) montre comment, en l'absence d'information sur leur devenir individuel et collectif (immédiat comme à long terme), les détenus se raccrochent aux " bembas ", "facteurs d'espoirs et aussi des peurs, mais avant tout moyens naturellement artisanaux pour comprendre la désinformation et l'incertitude ". Si la rumeur doit être appréhendée comme un triple mouvement de production, circulation et réception des discours ( $/$ bid.), elle doit, conjointement se lire comme rapport - voire un rapport de force - à l'institution.

Dans le cas présent, l'incertitude sur le devenir (transfert sur la péninsule ibérique ou refoulement au pays d'origine) est accentuée par des procédures de réadmission assez opaques entre les gouvernements espagnols et sénégalais (Pian, 2009b) donnant lieu certes, à des vagues de refoulements, mais aussi parfois, comme durant l'été 2006, à plusieurs vagues d'acheminement des migrants en Espagne. Qui dit incertitude, dit aussi place donnée à la chance et au destin : dès lors que les règles du jeu institutionnelles semblent arbitraires, un espace d'opportunités est par-là même ouvert. Le défi lancé au " monde frontière " (Cuttitta, 2007) se fait alors à travers la circulation de légendes depuis les lieux de départ. Dans les banlieues de Dakar, le récit suivant - avec quelques variantes - $\mathrm{m}^{\prime}$ a été plusieurs fois conté ; je l'ai aussi entendu lors de veillées entre jeunes Sénégalais : un capitaine de pêche achemine une pirogue contenant une soixantaine de passagers jusqu'aux îles Canaries. En passe d'être refoulé, il avertit les Espagnols : "si vous me renvoyez au Sénégal, je vais revenir et j'amènerai encore plus de personnes ". Quelque temps plus tard, il parvient à nouveau aux îles Canaries accompagné du double de passagers. Il est à nouveau refoulé et réitère sa mise en garde. La troisième fois, il arrive aux îles Canaries avec trois fois plus de passagers. Les Espagnols n'osent plus le refouler et il est transféré en Espagne. Au-delà des variantes entendues ${ }^{26}$, ce récit comportant une intrigue et un dénouement fait référence à une actualité immédiate (les traversées en pirogue vers les îles Canaries) tout en étant empreint d'une moralité invitant à faire preuve de persévérance : car, si tout reste possible, c'est aussi que rien n'est véritablement joué d'avance, le cadre légal de la gestion des migrations apparaissant tout à la fois discrétionnaire et arbitraire.

26 Notamment en ce qui concerne le nombre de passagers. 


\section{Conclusion}

Bien que les terrains d'enquête dont il est ici question remontent à quelques années, les enjeux soulevés demeurent d'une profonde actualité : en témoignent, en février 2017, les tentatives de passages collectives des enclaves de Ceuta et Melilla par plusieurs centaines de migrants subsahariens. L'une des caractéristiques contemporaines des frontières - qu'elles soient terrestres ou maritimes - est le nombre de morts qu'elles produisent, comme en attestent les données fournies par I'OIM $(2014 ; 2016)$ ou de nombreuses ONG de défense des droits des migrants. Selon le point de vue d'où l'on se place, les frontières matérialisent et symbolisent tout à la fois inclusion protectrice ou exclusion mortifère. Dans le cours des expériences migratoires toutefois, il n'existe pas un registre de discours unique mettant en scène la mort, mais différentes orientations discursives selon les références du contexte d'énonciation. En s'intéressant aux " mises en voix des évènements vécus " (Canut et Sow, 2014 : 16), I'article a néanmoins tenté de mettre au jour des invariants et des similitudes dans les manières de dire ce qui est perçu comme violence aux frontières par ceux qui tentent de les franchir clandestinement. Si l'on considère le langage comme " activité de mises en formes" (Cassirer, 1972 : 30) produisant des "formes différentes de conception du Moi et du Monde "(Ibid. : 33), ces mises en mots renseignent sur la manière dont l'ordre (ou dés-ordre) institutionnel est perçu et vécu, engageant un rapport aux agents de l'État. S'intéresser aux mises en mots invite alors à saisir comment les frontières se forment, aussi, dans et par les discours. 


\section{Références bibliographiques}

Agamben Giorgio (1998) Homo Sacer : le pouvoir souverain et la vie nue, Le Seuil, Paris, $213 \mathrm{p}$.

Agier Michel (2014) Parcours dans un paysage flottant de frontières, Revue Européenne des Migrations Internationales, 30 (2), pp. 13-23.

Agier Michel (2013) La condition cosmopolite. L'anthropologie à l'épreuve du piège identitaire, Paris, La découverte, $211 \mathrm{p}$.

Aldrin Philippe (2005) Sociologie politique des rumeurs, Paris, Puf, 289 p.

Amilhat-Szary Anne-Laure (2015) Qu'est-ce qu'une frontière aujourd'hui, Paris, Puf, 164 p.

Amilhat-Szary Anne-Laure and Giraut Frédéric (Dirs.) (2015) Borderities and the Politics of contemporar mobile border, New York, Palgrave Macmilan, 324 p.

Andersson Ruben (2014) Time and the migrant other: European border controls and the temporal economics of illegality, American Anthropologist, 116 (4), pp. 1-15.

Beaud Stéphane (1996) L'usage de l'entretien en sciences sociales. Plaidoyer pour " I'entretien ethnographique ", Politix, 9 (35), pp. 226-257.

Beaud Stéphane et Weber Florence (2003) Guide de l'enquête de terrain : produire et analyser des données ethnographiques, Paris, La découverte, 357 p.

Bliber Coutin Susan (2003) Illegality, borderlands and Space of Nonexistence, in Richard Warren Perry and Bill Maurer Dirs., Globalization under construction: Governementality, Law and Identity, London, University of Minesota Press Mineapolis, pp. 171-202.

Boe S. Carolina (2015) The Undeported/Les non expulsés. Foreigns National Caughts Between Criminal Justice and Immigration Enforcement in France and the US, Thèse de doctorat d'anthropologie, Université de Aarhus, Danemark.

Bonhomme Julien (2009) Les voleurs de sexe. Anthropologie d'une rumeur africaine, Paris, Seuil, $192 \mathrm{p}$.

Bouilly Emmanuelle (2008) La couverture médiatique du Collectif pour la lutte contre l'immigration clandestine de Thiaroye-sur-Mer (Dakar-Sénégal). Une mise en abîme du discours produit au "Nord " sur le "Sud ", Asylon(s), 3 [en ligne]. URL : http://www.reseau-terra.eu/article721.html

Brambilla Chiara (2014) Exploring the Critical Potential of the Borderscapes Concept, Geopolitics, 20 (1), pp. 14-34.

Canut Cécile et Sow Alioune (2014) Les voix de la migration. Discours, récits et productions artistiques, Cahiers d'études africaines, 213-214, pp. 9-25.

Cassirer Ernest (1972) La philosophie des formes symboliques. I. Le langage, Paris, Les Éditions de Minuit, $360 \mathrm{p}$.

Cefai Daniel (2001) Les cadres de I'action collective, in Daniel Cefai et Danny Trom Dirs., Les formes de l'action collective. Mobilisations dans des arènes publiques, Paris, Ehess, pp. 51-98.

Clochard Olivier (2016) Révoltes, protestations et résistances du quotidien. Des étrangers à l'épreuve de la détention, Migrations Société, 28 (164) pp. 57-72. 
Clochard Olivier (2014) L'enfermement des étrangers à travers le prisme des papiers, Champ pénal/Penal field, 11, [en ligne] consulté le 21/08/2016. URL : http://journals.openedition.org/champpenal/8858

Cusco Andrei (2016) A Contested Borderlands, Budapest, Central European University Press, $420 \mathrm{p}$.

Cuttitta Paolo (2015) La frontière Lampedusa. Mise en intrigue du sécuritaire et de I'humanitaire, Cultures \& Conflits, 99-100, pp. 99-115.

Cuttitta Paolo (2007) Le monde-frontière. Le contrôle de l'immigration dans I'espace globalisé, Cultures \& Conflits, 68, pp. 61-84.

Darley Mathilde (2009) L'enfermement des étrangers en Autriche et en République tchèque : du contrôle et de son contournement, in Carolina Kobelinsky et Chowra Makaremi Dirs., Enfermés dehors. Enquêtes sur le confinement des étrangers, Paris, Éditions du Croquant, pp. 63-83.

De Genova Nicholas (2005) Working the Boundaries: Race, Space, and Mexican "Illegality" in Mexican Chicago, Durham, Duke University Press, 352 p.

De Ípola Emilio (2006) La bemba. Vie et mort des rumeurs dans une prison politique (Argentine, 1976-1983), Diogène, 1 (213), pp. 174-201.

Dubar Claude (1991) La socialisation, Paris, Armand Colin, 255 p.

Fassin Didier (2011) Policing Borders, Producing Boundaries.The Governmentality of Immigration in DarkTimes, Annual Review of anthropology, 40, pp. 213-226.

Fassin Didier (2010) La raison humanitaire. Une histoire morale du temps présent, Paris, Seuil/Gallimard, $358 \mathrm{p}$.

Ferrer-Gallardo Xavier, Albet-Mas Abdel and Espineica Keina (2016) Euro-African invisibilisations in the border(land)scape of PuntaTarifa, in Chiara Brambilla, Jussi Laine, James W. Scott and Gianluca Bocchi Dirs., Borderscaping: Imaginations and pratices of border making, New York, Routledge, pp. 123-130.

Fischer Nicolas (2005) Clandestins au secret. Contrôle et circulation de l'information dans les centres de rétention administrative français, Cultures \& Conflits, 57, [en ligne] consulté le 27/12/2015. URL : http://journals.openedition.org/ conflits/1739

Fischer Nicolas et Spire Alexis (2009) L'État face aux illégalismes, Politix, 3 (87), pp. 7-20.

Foucher Michel (1998) Fronts et frontières. Un tour du monde géopolitique, Paris, Fayard, $691 \mathrm{p}$

Foucher Vincent (2003) Pas d'alternance en Casamance ? Le nouveau pouvoir sénégalais face à la revendication séparatiste casamançaise, Politique africaine, $3(91)$, pp. 101-119.

Goffman Erving (1968) Asiles : études sur la condition sociale des malades mentaux et autre reclus, Paris, Éditions de Minuit, $452 \mathrm{p}$.

Green Sarah (2012) A sens of border, in Thomas M. Wilson and Hasting Donnan Dirs., A Companion to Border Studies, Oxford, Wiley-Blackwell, pp. 573-592.

Griffiths Melanie (2014) Out of Time: The Temporal Uncertainties od Refused Asylum Seekers and Immigration Detainees, Journal of Ethnic and Migration Studies, 40 (12), pp. 1991-2009. 
Ípola Emilio (de) (2006) La Bemba. Vie et mort des rumeurs dans une prison politique (Argentine, 1976-1983), Diogène, 213 (1), pp. 174-201.

Lask Tomke (1995) Les professionnels de la frontière : entretien avec trois douaniers français, Quaderni. Penser la frontière, 27, pp. 115-121.

Lecadet Clara (2012) Tinzawaten c'est le grand danger pour nous les immigrés, Hermès, 63, pp. 95-100.

Leclerc-Olive Michèle (1997) Le dire de l'événement (biographique), Villeneuve d'Ascq, Presse du septentrion, 260 p.

Lochak Danièle (1985), Étrangers de quels droits ?, Paris, Puf, 256 p.

Makaremi Chowra (2009) Violence et refoulement dans la zone d'attente de Roissy, in Carolina Kobelinsky et Chowra Makaremi Dirs., Enfermés dehors. Enquêtes sur le confinement des étrangers, Paris, Éditions du Croquant, pp. 41-62.

Makaremi Chowra et Kobelinsky Carolina (2009) Introduction, in Carolina Kobelinsky et Chowra Makaremi Dirs., Enfermés dehors. Enquêtes sur le confinement des étrangers, Paris, Éditions du Croquant, pp. 11-23.

Marcus George E. (2010) Ethnographie du/dans le système-monde. L'émergence d'une ethnographie multisituée, in Daniel Cefaï Dir., L'engagement ethnographique, Paris, Ehess, pp. 371-398.

Massamba Fiston (2006) Mouvements aux frontières. Rabat, chronique d'une mobilisation, Propos recueillis par Claire Rodier, Vacarme, 37, [en ligne] consulté le 20/08/2016. URL : http://www.vacarme.org/article1188.html

Médecins Sans Frontières (2013) Violences, vulnérabilités et migration : bloqués aux portes de l'Europe. Un rapport sur les migrants subsahariens en situation irrégulière au Maroc, 42 p., [en ligne] consulté le 29/08/2016. URL : http://www2. ohchr.org/english/bodies/cmw/docs/ngos/MSF_Morocco18_fr.pdf

Médecins Sans Frontières (2005) Violence et immigration : rapport sur l'immigration d'origine subsaharienne en situation irrégulière au Maroc, [en ligne] consulté le 29/08/2016. URL : https://www.msf.fr/sites/www.msf.fr/files/2005-0929-MSFE.pdf

Moulard-Kouka Sophie (2014) Le regard entre deux rives : la migration et l'exil dans le discours des rappeurs sénégalais, Cahiers d'études africaines, 213-214, pp. 415-449.

OIM (2016) L'OIM recense 3771 décès de migrants dans la Méditerranée en 2015, [en ligne] consulté le 02/09/2016. URL : https://www.iom.int/fr/news/loimrecense-3-771-deces-de-migrants-dans-la-mediterranee-en-2015

OIM (2014) Fatal Journeys: Tracking Lives Lost During Migration, Geneva, IOM, 216 p., [online] last checked on 30/08/2016. URL: http://www.iom.int/ files/live/sites/iom/files/pbn/docs/Fatal-Journeys-Tracking-Lives-Lost-duringMigration-2014.pdf

Petit Véronique, Pizzolato Giulia et Ly Mohamed (2014) La migration face à la santé mentale au Sénégal : récits, discours et imaginaires, in Cécile Canut et Catherine Mazuric Dirs., La migration prise aux mots. Mise en récits et en image des migrations transafricaines, Paris, Le cavalier Bleu, pp. 225-242.

Pian Anaïk (2016) D'une Europe à l'autre, une vie faite de frontières, Migrations Société, 28 (164), pp. 135-150. 
Pian Anaïk (2013) Des frontières de la migration aux représentations de l'Europe, Hommes et Migrations, 1304, pp. 11-17.

Pian Anaïk (2011a) Migrations internationales au prisme des rapports familiaux. Les familles sénégalaises à l'épreuve des refoulements des îles Canaries, Revue Européenne des Migrations Internationales, 27 (2), pp. 77-100.

Pian Anaïk (2011b) The "discursive framework" of development and the repertoire of actions of Senegalese deportee associations, in Jocelyne Streiff-Fenart and Aurelia Wa Kabwe Segatti Dirs., The challenge of the threshold. Border closures and migration movements in Africa, Maryland, Lexington books, pp. 57-70.

Pian Anaïk (2010) Trajectoires de rapatriés. Éléments pour une réflexion en termes d'expérience, Hommes et Migrations, 1286-1287, pp. 74-97.

Pian Anaïk (2009a) Aux nouvelles frontières de l'Europe. L'aventure incertaine des Sénégalais au Maroc, Paris, La Dispute, 237 p.

Pian Anaïk (2009b) Face aux enjeux politiques locaux, aide au développement en échange de retours forcés : le Plan Reva au Sénégal, in Olivier Clochard Dir., Atlas des migrants en Europe. Géographie critique des politiques migratoires, Paris, Armand Colin, pp. 88-90.

Puig Nicolas, Bontemps Véronique et Hily Marie-Antoinette (Coords.) (2014) Composer (avec) la frontière. Passages, parcours migratoires et échanges sociaux, Revue Européenne des Migrations Internationales, 30 (2), pp. 7-168.

Rajaram Prem Kumar and Grundy-Warr Carl (Dirs.) (2008) Boderscapes. Hidden geographies and politics at territory's Edge, Minneapolis, The University of Minnesota Press, $330 \mathrm{p}$.

Ritaine Évelyne (Coord.) (2015) Effets-frontières en Méditerranée : contrôles et violences, Cultures \& Conflits, 99-100, pp. 11-142.

Séchet Raymonde et Veschambre Vincent (Dirs.) (2006) Penser et faire la géographie sociale, Rennes, PUR, $397 \mathrm{p}$.

Shindel Estela (2016) Bare life at the European borders. Entanglements of technology, society and nature, Journal of Borderlands Studies, 31 (2), pp. 219-234.

Tassin Louise (2013) D'une frontière à l'autre. Production et usages des catégories de l'altérité dans un centre de rétention, Hommes et Migrations, 1304, pp. 51-58.

Timera Mahamet (2001) Les migrations des jeunes Sahéliens : affirmation de soi et émancipation, Autrepart, 18, pp. 37-49. 


\section{Anaïk Pian}

\section{Les espaces discursifs de la frontière : mort et arbitraire dans le voyage vers l'Europe}

En mobilisant différents terrains menés auprès de Sénégalais cherchant à rejoindre clandestinement l'Europe, l'article s'intéresse à la fabrique des discours sur la mort et, partant, sur la violence aux et des frontières. Une première partie met en perspective différents registres de discours sur la mort, registres faisant référence à une mort physique au sens propre ainsi qu'à une mort sociale au sens figuré. Les discours évoquant la mort ou le risque de mort dans ces routes vers l'Europe comportent toutefois de profondes nuances selon qu'il s'agisse de légitimer, justifier voire de rationnaliser l'émigration, de faire part d'expériences extrêmes confrontant aux obstacles naturels ou de décrire les formes de violences institutionnelles vécues dans le cours du périple effectué. La seconde partie s'intéresse à la manière dont la confrontation à une gestion institutionnelle opaque des migrations - notamment en centre de rétention alimente la production et circulation de rumeurs, qui peuvent être vues, en partie, comme un mode de résistance à un contexte d'incertitude. S'intéresser aux mises en mots invite alors à se demander si les frontières ne se forment pas, aussi, dans et par les discours.

\section{Discursives Spaces of Border: Death and Arbitrariness in Journeys towards Europe}

By mobilizing different field studies carried on with Senegalese trying to get to Europe clandestinely, this article examines the form and content of narratives about death during border crossing, and, drawing on this, about violence of and at borders. A first part puts into perspective different registers in narratives about death, referring to physical death in a literal sense as well as social death in a figurative sense. However deep nuances may be found in narratives evoking death or death risk in these migration routes towards Europe, depending on whether they legitimate, justify or even rationalise migration, and also depending whether they relate extreme experiences confronting to natural obstacles, or describe forms of institutional violence experienced in the course of the journey. The second part shows how confrontation with an opaque management of migrations - particularly in holding centers - contributes to the start and spreading of rumours, which can be partly seen as a form of resistance to a context of uncertainty. Examining these narratives raises the question whether borders are also formed by and through discourses. 


\section{Espacios discursivos de la frontera: la muerte y lo arbitrario en viajes hacia Europa}

Movilizando diferentes trabajos de campo llevados a cabo entre senegaleses que buscan llegar de forma clandestina a Europa, el artículo examina la fábrica de discursos sobre la muerte y la violencia en y de las fronteras. La primera parte pone en perspectiva diferentes registros de discurso sobre la muerte; registros que se refieren a una muerte física en el sentido estricto y a una muerte social en el sentido figurado. Los discursos que evocan la muerte o el riesgo de muerte en las rutas hacia Europa conllevan sin embargo profundos matices según se trate de legitimar, justificar o incluso racionalizar la emigración, de dar cuenta de experiencias extremas confrontándose a obstáculos naturales o de describir las formas de violencias institucionales vividas en el transcurso del periplo. La segunda parte examina la manera en que la confrontación con una gestión institucional opaca de las migraciones - sobre todo en los centros de internamiento - alimenta la producción y la circulación de rumores que parcialmente pueden ser vistos como un modo de resistencia en un contexto de incertidumbre. Interesarse por las formas en que ello se pone en palabras invita a preguntarse si las fronteras no se forman, también, en y a través de los discursos. 